\title{
EVALUATING CONSERVATION STATUS, THREATS AND POPULATION TRENDS OF LEUCADENDRON, A PLANT GENUS ENDEMIC TO THE CAPE REGION IN SOUTH AFRICA
}

\author{
Samuel O. BAMIGBOYE \\ Department of Plant Sciences, Olabisi Onabanjo University, 2002, Nigeria \\ e-mail: reachtoba@gmail.com
}

\begin{abstract}
Declines in endemic species have significant impact on global biodiversity loss. More efforts need to be harnessed to further protect endemic species from the current global extinction crisis. This study evaluated the current conservation status, factors responsible for risk of extinction and the trends in populations of Leucadendron, a plant genus endemic to the Cape region in South Africa. The SANBI (South African National Biodiversity Institute) Red List was employed in this study. The results revealed that over $50 \%$ of taxa in this genus are threatened and most of the species are exposed to habitat destruction and the presence of invasive species. It was also discovered that two species of this genus are currently extinct, which implies this genus is facing a high risk of extinction. More efforts, such as designing effective methods of controlling forces responsible for the risk of extinction of taxa in this genus, should be put in place to prevent their complete extirpation in future.
\end{abstract}

Keywords: Conservation, endemism, extinction risk, population decline, threatened species

\section{Introduction}

Species extinction is currently a global problem and it is translating into loss of ecosystem services [1]. The current decline in species populations puts Earth's biodiversity at future risk [2], which makes continuous review of species that are at risk of extinction extremely important. Threatened species need continuous evaluation, at intervals of at least a decade, to keep track of changes in their conservation status and to determine whether efforts made in conservation of threatened species are generating the results required [3]. One of the steps to evaluate extinction risk of taxa is to determine the factors that are responsible for that risk and recommend how the effect of these ecological forces can be mitigated [4].

Endemic species occur exclusively within certain geographical areas [5]. They are highly vulnerable to extinction due to anthropogenic threats, small population sizes, specific habitat conditions, short reproduction capacity and population decline [6]. Consistent evaluation of the conservation status of endemic species is important in order to keep track of increase in their extinction risk and also to determine their response to conservation actions put in place to reduce that risk [7].

The genus Leucadendron belongs to the family Proteaceae [8]. Endemic to the Cape region of South Africa [9], this genus consists of short and tall evergreen shrubs [10]. Some of the species are widespread while others are rare. Their flowers possess distinct coloured petal-like bracts [11] and the majority of them are used as cut flowers [12].

This study evaluated the conservation status, threats and population trends of 
Leucadendron using the SANBI Red List. This is to show the current degree of extinction risk in the genus and indicate factors that should be controlled to reduce the risk of extinction of this genus.

\section{Methodology}

The South African National Biodiversity Institute (SANBI) is the national conservation body in South Africa charged with the responsibility of assessing plant and animal species in South Africa for conservation purposes. The SANBI Red List contains the conservation status of South African plant taxa. It also includes information such as threats, uses, trends in population and other relevant information that reveals the state of conservation of taxa listed on the Red List. IUCN guideline are strictly adhered to by SANBI in evaluating the conservation status of South African flora and fauna and this has made the SANBI Red List a globally accepted platform for determining the status of plant and animal taxa that occur in South Africa. The 2020 version of the Red List [13] was employed in this study to evaluate the current conservation status of the genus Leucadendron. The following were determined from the Red List: Conservation status, threats and population trends of Leucadendron species. Percentages of taxa under different SANBI Red List categories for Leucadendron were calculated and the percentages of taxa under different categories of population trend (increasing, decreasing, stable, fluctuating) were determined. The percentages of taxa in this genus facing different categories of threats were calculated.

\section{Results}

A total of 96 recognized species and subspecies of Leucadendron were found on the SANBI Red List. The following are the percentages of taxa in Leucadendron that fall under different SANBI Red List Categories: $2 \%$ are already Extinct (EX), $18.8 \%$ are Critically Endangered (CE), $18.8 \%$ are Endangered (EN), 13.5\% are Vulnerable (VU), 17.7\% are Near Threatened (NT), 2\% are Rare, $1 \%$ are Data Deficient Taxonomically Problematic (DDT) and 26\% are of the Least Concern (LC); 51.1\% are threatened (Critically Endangered + Endangered + Vulnerable) and $71.8 \%$ are of conservation concern (Critically Endangered + Endangered + Vulnerable + Near Threatened + Rare + Data Deficient Taxonomically Problematic). The percentages of taxa in Leucadendron threatened by different kinds of ecological forces are as follows: $66.7 \%$ are threatened by habitat destruction, $60.4 \%$ are threatened by the presence of invasive species, $23 \%$ are threatened by fire occurrence, $17.7 \%$ are threatened by pollution, $16.7 \%$ are threatened by harvesting, $4.1 \%$ are threatened by grazing, $2 \%$ threatened by drought, $1 \%$ threatened by pest control, $1 \%$ threatened by reproductive failure, $10.4 \%$ are not threatened and the factors threatening $9.4 \%$ that are of conservation concern are unknown. The percentage of taxa experiencing population decrease in this genus is $62.5 \%$ and the populations of $27 \%$ of taxa are stable. Those that are fluctuating between decreasing and stable are $5.2 \%$, and the population trends of $3 \%$ are unknown.

\section{Discussion}

Conservation status changes over time, with species becoming either more threatened or less threatened due to conservation interventions [3]. Thus it is important to determine current conservation status of threatened plant groups regularly. Because Leucadendron has two species that are currently extinct (Figure1; Table 1), 51.1\% currently threatened (Figure 1; Table 1) and 
$71.8 \%$ of conservation concern (Table 1), it can be concluded that this genus is facing a high risk of extinction.

Table 1: Listing of the species in the genus Leucadendron, their SANBI Red List Status and the threats each is facing

\begin{tabular}{|c|c|c|c|}
\hline Species & $\begin{array}{l}\text { SANBI Red } \\
\text { List Status }\end{array}$ & Population trend & Threats \\
\hline $\begin{array}{l}\text { Leucadendron album (Thunb.) } \\
\text { Fourc. }\end{array}$ & $\mathrm{LC}$ & Stable & $\begin{array}{l}\text { Fire occurrences, invasive species } \\
\text { presence }\end{array}$ \\
\hline $\begin{array}{l}\text { Leucadendron arcuatum (Lam.) } \\
\text { I.Williams }\end{array}$ & $\mathrm{LC}$ & Stable & Habitat destruction \\
\hline $\begin{array}{l}\text { Leucadendron argenteum (L.) } \\
\text { R.Br. }\end{array}$ & $\mathrm{EN}$ & Decreasing & $\begin{array}{l}\text { Habitat destruction, invasive } \\
\text { species presence }\end{array}$ \\
\hline Leucadendron barkerae I.Williams & $\mathrm{LC}$ & Stable & Fire occurrences \\
\hline Leucadendron bonum I.Williams & $\mathrm{CE}$ & Decreasing & $\begin{array}{l}\text { Fire occurrences, reproductive } \\
\text { failure }\end{array}$ \\
\hline $\begin{array}{l}\text { Leucadendron brunioides Meisn. } \\
\text { var. brunioides }\end{array}$ & $\mathrm{LC}$ & Stable & No threats \\
\hline $\begin{array}{l}\text { Leucadendron brunioides Meisn. } \\
\text { var. flumenlupinum I.Williams }\end{array}$ & $\mathrm{CE}$ & Decreasing & $\begin{array}{l}\text { Pollution, invasive species } \\
\text { presence, habitat destruction }\end{array}$ \\
\hline $\begin{array}{l}\text { Leucadendron burchellii } \\
\text { I.Williams }\end{array}$ & NT & Stable & $\begin{array}{l}\text { Invasive species presence, habitat } \\
\text { destruction }\end{array}$ \\
\hline Leucadendron cadens I.Williams & Rare & Stable & No threat \\
\hline $\begin{array}{l}\text { Leucadendron chamelaea (Lam.) } \\
\text { I.Williams }\end{array}$ & $\mathrm{CE}$ & Decreasing & $\begin{array}{l}\text { Invasive species presence, habitat } \\
\text { destruction, pest control }\end{array}$ \\
\hline $\begin{array}{l}\text { Leucadendron cinereum (Sol. ex } \\
\text { Aiton) R.Br. }\end{array}$ & VU & Decreasing & $\begin{array}{l}\text { Invasive species presence, habitat } \\
\text { destruction, pollution, }\end{array}$ \\
\hline $\begin{array}{l}\text { Leucadendron comosum (Thunb.) } \\
\text { R.Br. subsp. comosum }\end{array}$ & LC & Stable & No threat \\
\hline $\begin{array}{l}\text { Leucadendron comosum (Thunb.) } \\
\text { R.Br. subsp. homaeophyllum } \\
\text { (Meisn.) I.Williams }\end{array}$ & $\mathrm{CE}$ & Decreasing & $\begin{array}{l}\text { Harvesting, fire occurrences, } \\
\text { invasive species presence }\end{array}$ \\
\hline $\begin{array}{l}\text { Leucadendron concavum } \\
\text { I.Williams }\end{array}$ & $\mathrm{EN}$ & Unknown & Fire occurrences \\
\hline $\begin{array}{l}\text { Leucadendron conicum (Lam.) } \\
\text { I.Williams }\end{array}$ & NT & Decreasing & Unknown \\
\hline $\begin{array}{l}\text { Leucadendron coniferum (L.) } \\
\text { Meisn. }\end{array}$ & VU & Decreasing & $\begin{array}{l}\text { Harvesting, invasive presence, } \\
\text { habitat destruction, pollution }\end{array}$ \\
\hline Leucadendron cordatum E.Phillips & Rare & Stable & $\begin{array}{l}\text { Habitat destruction, fire } \\
\text { occurrences }\end{array}$ \\
\hline $\begin{array}{l}\text { Leucadendron coriaceum } \\
\text { E.Phillips \& Hutch. }\end{array}$ & $\mathrm{EN}$ & Decreasing & $\begin{array}{l}\text { Habitat destruction, invasive } \\
\text { species presence, pollution }\end{array}$ \\
\hline $\begin{array}{l}\text { Leucadendron corymbosum } \\
\text { P.J.Bergius }\end{array}$ & VU & Decreasing & $\begin{array}{l}\text { Pollution, invasive species } \\
\text { presence, habitat destruction }\end{array}$ \\
\hline $\begin{array}{l}\text { Leucadendron crassulaefolium } \\
\text { (Salisb. ex Knight) I.Williams }\end{array}$ & DDT & Stable & Unknown \\
\hline $\begin{array}{l}\text { Leucadendron cryptocephalum } \\
\text { Guthrie }\end{array}$ & $\mathrm{EN}$ & Decreasing & $\begin{array}{l}\text { Habitat destruction, invasive } \\
\text { species presence, overgrazing }\end{array}$ \\
\hline $\begin{array}{l}\text { Leucadendron daphnoides } \\
\text { (Thunb.) Meisn. }\end{array}$ & EN & Decreasing & $\begin{array}{l}\text { Habitat destruction, invasive } \\
\text { species presence }\end{array}$ \\
\hline $\begin{array}{l}\text { Leucadendron diemontianum } \\
\text { I.Williams }\end{array}$ & NT & Stable & No threat \\
\hline $\begin{array}{l}\text { Leucadendron discolor E.Phillips } \\
\text { \& Hutch. }\end{array}$ & $\mathrm{EN}$ & Decreasing & Habitat destruction, harvesting \\
\hline $\begin{array}{l}\text { Leucadendron dregei E.Mey. ex } \\
\text { Meisn. }\end{array}$ & $\mathrm{EN}$ & Decreasing & Fire occurrence \\
\hline $\begin{array}{l}\text { Leucadendron dubium (H.Buek ex } \\
\text { Meisn.) E.Phillips \& Hutch. }\end{array}$ & NT & Decreasing & Habitat destruction \\
\hline $\begin{array}{l}\text { Leucadendron elimense E.Phillips } \\
\text { subsp. elimense }\end{array}$ & $\mathrm{EN}$ & Decreasing & $\begin{array}{l}\text { Invasive species presence, } \\
\text { harvesting, habitat destruction }\end{array}$ \\
\hline
\end{tabular}


Leucadendron elimense E.Phillips subsp. salteri I.Williams

Leucadendron elimense E.Phillips subsp. vyeboomense I.Williams

Leucadendron ericifolium R.Br.

Leucadendron eucalyptifolium

H.Buek ex Meisn.

Leucadendron flexuosum

I.Williams

Leucadendron floridum R.Br.

Leucadendron foedum I.Williams

Leucadendron galpinii E.Phillips

\& Hutch.

Leucadendron gandogeri Schinz

ex Gand.

Leucadendron glaberrimum

(Schltr.) Compton subsp.

erubescens I.Williams

Leucadendron glaberrimum

(Schltr.) compton subsp.

glaberrimum

Leucadendron globosum (Kenn. ex

Andrews) I.Williams

Leucadendron grandiflorum

(Salisb.) R.Br.

Leucadendron gydoense I.Williams

Leucadendron immoderatum

Rourke

Leucadendron lanigerum H.Buek

ex Meisn. var. laevigatum Meisn.

Leucadendron lanigerum H.Buek

ex Meisn. var. lanigerum

Leucadendron laureolum (Lam.)

Fourc.

Leucadendron laxum I.Williams

Leucadendron levisanus (L.)

P.J.Bergius

Leucadendron linifolium (Jacq.)

R.Br.

Leucadendron loeriense I.Williams

Leucadendron loranthifolium

(Salisb. ex Knight) I.Williams

Leucadendron macowanii

E.Phillips

Leucadendron meridianum

I.Williams

Leucadendron meyerianum

H.Buek ex E.Phillips \& Hutch.

Leucadendron microcephalum

(Gand.) Gand. \& Schinz
LC

EN

CE

Decreasing

Decreasing

Stable

Decreasing

CE

Decreasing

CE

Decreasing

Decreasing

LC

LC

LC

CE

EX

EN

CE

CE

EN

Decreasing

Stable

Stable

Stable

Decreasing

Not Applicable

Decreasing

Fluctuating

Fluctuating

Decreasing

Unknown

Decreasing

Decreasing

VU

LC

NT

CE

NT

EN

LC
Decreasing

Stable

Decreasing

Fluctuating

Decreasing

Decreasing

Unknown
Habitat destruction, invasive species occurrence, harvesting Habitat destruction, invasive species presence, fire occurrences, Habitat destruction, invasive species occurrence, fire occurrences Habitat destruction, invasive species occurrence Habitat destruction, invasive species occurrence Habitat destruction, harvesting, invasive species presence Habitat destruction, invasive species presence, pollution Invasive species presence, harvesting, habitat destruction Invasive species presence, harvesting, fire occurrences No threats

No threats

Invasive species presence, Habitat destruction

Unknown

Habitat destruction

Habitat destruction, invasive species presence

Habitat destruction, invasive species presence, pollution Habitat destruction, invasive species presence, pollution, harvesting

Habitat destruction, invasive species presence

Pollution, invasive species presence, harvesting, habitat destruction

Habitat destruction, pollution, invasive species presence,

Invasive species presence, habitat destruction, pollution, harvesting Habitat destruction, fire occurrence, invasive species presence,

Habitat destruction

Habitat destruction, harvesting, invasive species presence Habitat destruction, invasive species presence Fire occurrences

Habitat destruction, invasive species presence 
Leucadendron modestum

I.Williams

Leucadendron muirii E.Phillips

Leucadendron nervosum E.Phillips

\& Hutch.

Leucadendron nitidum H.Buek ex $\quad$ LC

Meisn.

Leucadendron nobile I.Williams LC

Leucadendron olens I.Williams NT

Leucadendron orientale I.Williams EN

Leucadendron osbornei Rourke LC

Leucadendron platyspermum $\mathrm{R}$.Br. VU

Leucadendron pondoense A.E.van VU

Wyk

Leucadendron procerum (Salisb. VU

ex Knight) I.Williams

Leucadendron pubescens R.Br. LC

Leucadendron NT

pubibracteolatum I.Williams

Leucadendron radiatum E.Phillips EN

\& Hutch.

Leucadendron remotum I.Williams EN

Leucadendron roodii E.Phillips EN

Leucadendron rourkei I.Williams LC

Leucadendron rubrum Burm.f.

LC

Leucadendron salicifolium

(Salisb.) I.Williams

Leucadendron salignum

P.J.Bergius

Leucadendron sericeum (Thunb.)

R.Br.

Leucadendron sessile R.Br.

Leucadendron sheilae I.Williams

LC

LC

CE

NT

$\mathrm{VU}$

Leucadendron singulare I.Williams VU

Leucadendron sorocephalodes NT

E.Phillips \& Hutch.

Leucadendron spirale (Salisb. ex EX

Knight) I.Williams

Leucadendron spissifolium (Salisb. LC ex Knight) I.Williams subsp.

fragrans I.Williams

Leucadendron spissifolium (Salisb. NT

ex Knight) I.Williams subsp.

natalense (Thode \& Gilg)

I.Williams

Leucadendron spissifolium (Salisb. VU

ex Knight) I.Williams subsp.

oribinum I.Williams

Leucadendron spissifolium (Salisb. LC ex Knight) I.Williams subsp.

phillipsii (Hutch.) I.Williams
Decreasing

Decreasing

Decreasing

Stable

Stable

Stable

Decreasing

Stable

Decreasing

Decreasing

Decreasing

Decreasing

Fluctuating

Decreasing

Decreasing

Decreasing

Stable

Stable

Decreasing

Decreasing

Decreasing

Decreasing

Decreasing

Decreasing

Decreasing

Not Applicable

Stable

Decreasing

Decreasing

Stable
Invasive species presence, habitat destruction, pollution

Habitat destruction, invasive species presence, fire occurrences Unknown

No threat

No threat

Unknown

Invasive species presence, habitat destruction

No threat

Harvesting, invasive species presence, fire occurrences

Fire occurrences, habitat

destruction

Invasive species presence, habitat destruction

Habitat destruction, fire occurrences

Invasive species presence, habitat destruction

Fire occurrences

Fire occurrences, climate change Habitat destruction, fire occurrences, drought

No threat

Habitat destruction, invasive species presence

Habitat destruction, invasive presence, drought

Habitat destruction, grazing, invasive species presence

Habitat destruction, fire occurrences

Unknown

Invasive species presence, habitat destruction, pollution

Habitat destruction, pollution, invasive species presence

Unknown

Habitat destruction, invasive species presence

Habitat destruction, grazing

Unknown

Habitat destruction, invasive species presence

Habitat destruction, invasive species presence 
Leucadendron spissifolium (Salisb. LC ex Knight) I.Williams subsp. spissifolium

Leucadendron stellare (Sims)

Sweet

Leucadendron stelligerum

I.Williams

Leucadendron strobilinum (L.)

Druce

Leucadendron teretifolium

(Andrews) I.Williams

Leucadendron thymifolium (Salisb.

ex Knight) I.Williams

Leucadendron tinctum I.Williams

Leucadendron tradouwense

EN

Stable

I.Williams

Leucadendron uliginosum R.Br. NT subsp. glabratum I.Williams

Leucadendron uliginosum R.Br.

subsp. uliginosum

Leucadendron verticillatum

(Thunb.) Meisn.

Leucadendron xanthoconus

(Kuntze) K.Schum.

\section{NT}

VU

\section{CE Decreasing}

Stable

Decreasing

Decreasing

Decreasing

Decreasing

Decreasing

NT Decreasing

CE Fluctuating

LC Stable
Fire occurrences, invasive species presence

Habitat destruction, grazing, invasive species presence, fire occurrences

Habitat destruction, invasive species presence, pollution, harvesting

Fire occurrences

Habitat destruction, invasive species occurrence

Invasive species presence, habitat destruction, pollution

Unknown

Invasive species presence, harvesting, habitat destruction Habitat destruction, invasive species presence

Habitat destruction, invasive species presence

Habitat destruction, invasive species presence, harvesting Habitat destruction, invasive species presence

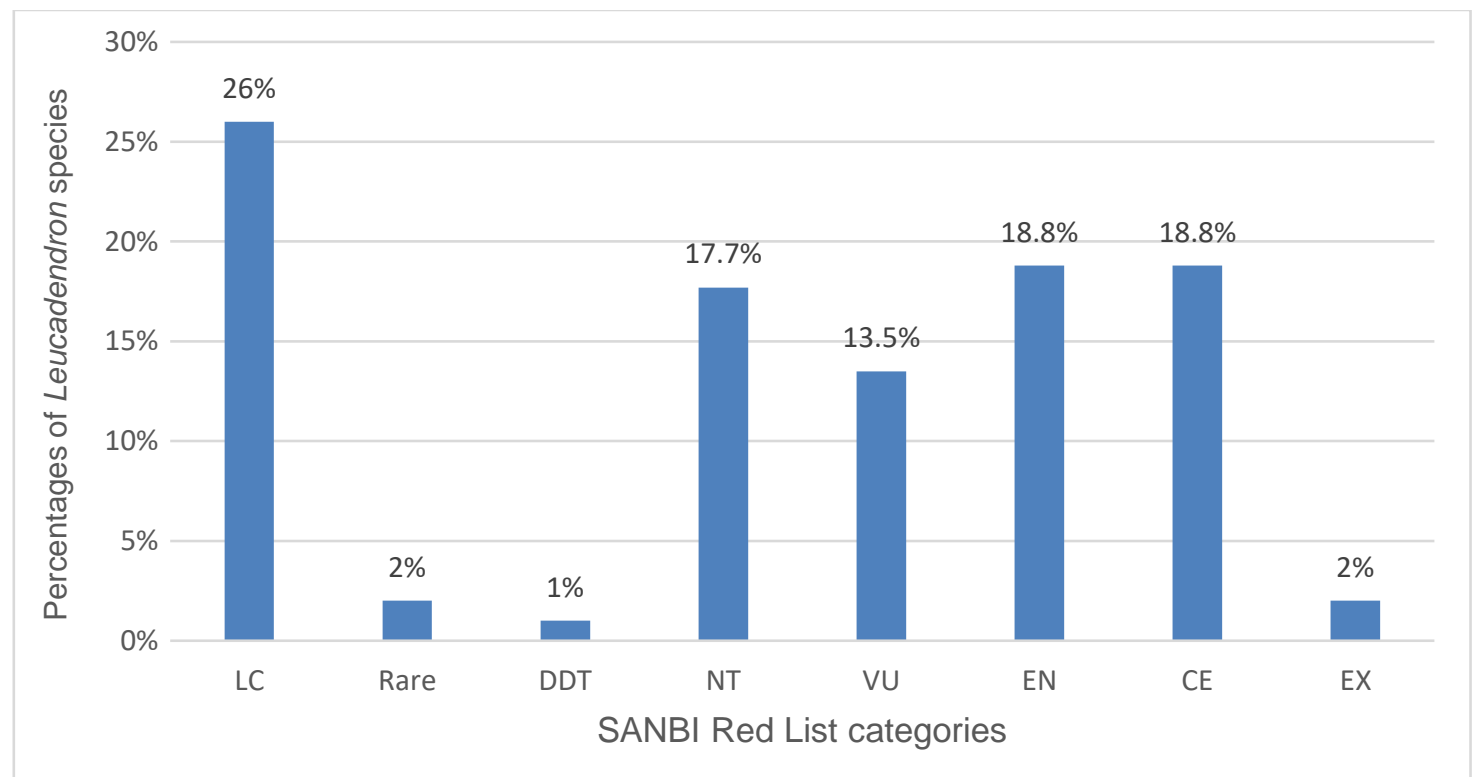

Fig. 1: Percentages of Leucadendron taxa that fall into different SANBI Red List categories

Certain factors are responsible for risk of extinction of threatened species [14] and these ecological forces need to be controlled to prevent further species decline and increase in biodiversity loss. This study recommends re-evaluation of Leucadendron species where their threats are listed as unknown (Table 1; Figure 2) to determine factors responsible for their risk of extinction. 


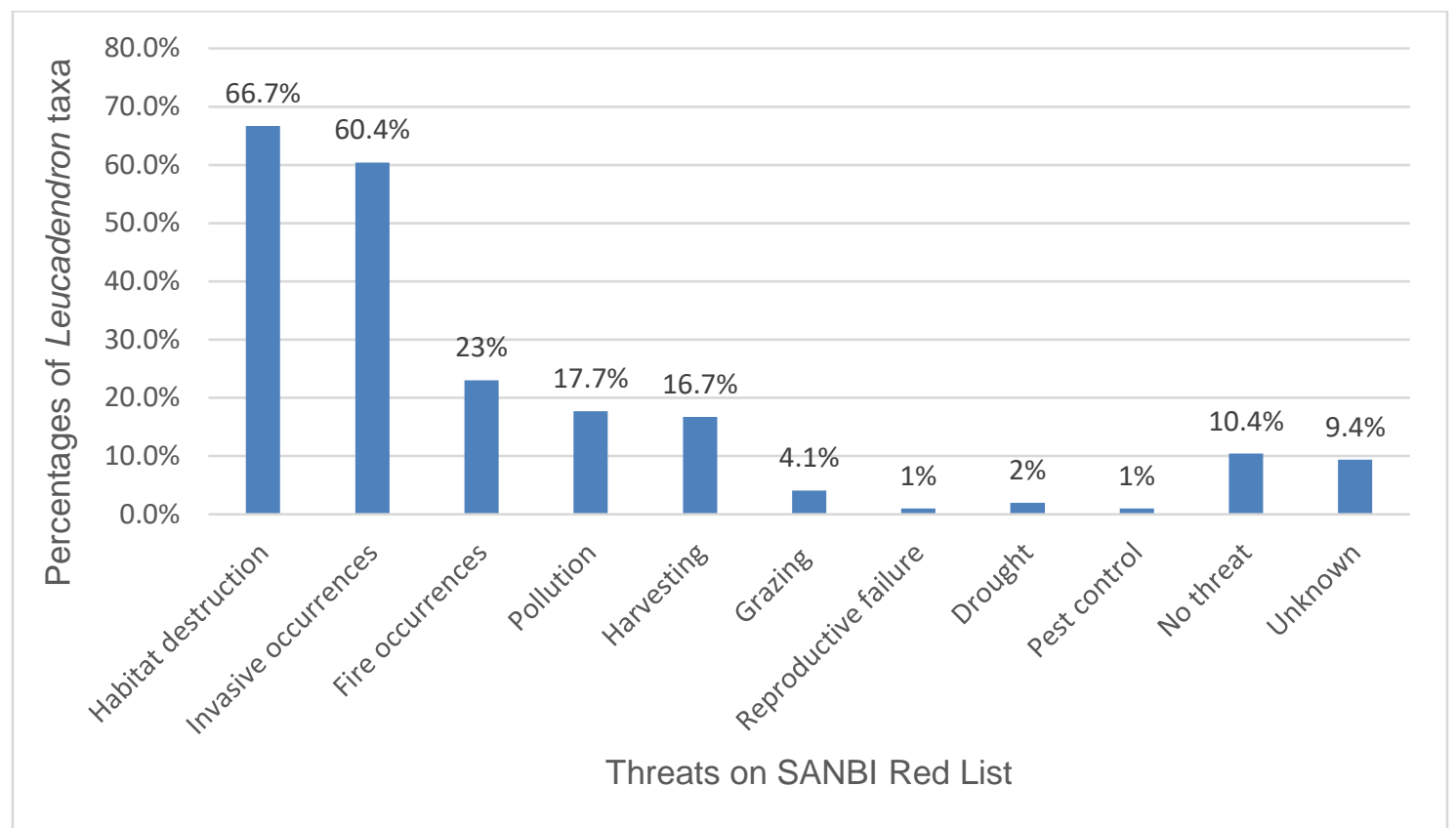

Fig. 2: Percentages of Leucadendron taxa that face different threats on the SANBI Red List

The trends in population size of species is one of the factors that determine risk of extinction of species [15]. Whether the population of threatened taxa is decreasing, increasing or stable will determine whether or not they will become more threatened in future [3]. The present study recommends re-evaluation of taxa whose population trends are unknown (Table 1) to determine which category of population trends they fall into. With a larger percentage of the taxa in the genus experiencing population decline (Table 1), it can be concluded that this genus is tending towards a higher extinction risk in the future. This implies that more proactive measures are needed to curtail the risk of extinction of this genus.

\section{Conclusion}

This study has shown that the genus Leucadendron, endemic to the Cape region of South Africa, is highly threatened and the factors responsible for the risk of extinction are revealed (Table 1). The study contributes to the body of literature that promotes advocacy for high level protection of endemic species because they the genus Leucadendron should be protected. Regeneration of these species should be encouraged to increase the number of populations.

\section{REFERENCES}

1. Leadley, P., Pereira, H.N., Alkemade, R. Fernandez-Manjarrés, J.F., Proença, V.,Scharlemann, J.P.W., Walpole, M.J., 2010, Biodiversity Scenarios: Projections of $21^{\text {st }}$ Century Change in Biodiversity and Associated Ecosystem Services. A Technical Report 28 for the Global Biodiversity Outlook 3, Technical Series No. 50, Secretariat of the Convention on Biological Diversity, Montreal, Canada.

2. Vamosi, J.C., Vamosi, S.M., 2008, Extinction risk escalates in the tropics, PLoS ONE, 3: e3886.

3. Bamigboye, S.O., Tshisikhawe P.M., Taylor, P.J., 2016, Review of extinction risk in African cycads, Phyton International Journal of Experimental Botany, 85 (1): 333-336.

4. Bamigboye, S.O., 2020, Evaluatng threats and conservation status of South African Aloe, Journal of Threatened Taxa, 12 (11): 16614-16619 htps://doi.org/10.11609/jot.5728.12.11.16614-16619. 
5. Burlakova, L.E., Karatayev, A.Y., Karatayev, V.A., May, M.E., Bennett, D.L., Cook, M.J., 2011, Endemic species: Contribution to community uniqueness, effect of habitat alteration, and conservation priorities, Biological Conservation, 144: 155-165.

6. Isik, K., 2011, Rare and endemic species: Why are they prone to extinction? Turkish Journal of Botany, 35: 411-417.

7. Moraswi I., Bamigboye, S.O., Tshisikhawe, P.M., 2019, Conservation status and threats to vascular plant species endemic to Soutpansberg Mountain in Limpopo Province, South Africa, International Journal of Plant Biology, 10: 7978: 14-16. htps://doi.org/10.4081/ pb.2019.7978

8. Douglas, A.W., 1995. Affinities, Flora of Australia, 16: 6-14.

9. Barker, N.P., Vanderpoorten, A., Morton, C.M., Rourke, J.P., 2004, Phylogeny, biography, and the evolution of life-history traits in Leucadendron (Proteaceae), Molecular Phylogenetic Evolution, 33: 845-860.

10. Thuller, W., Lavorel, S., Midgley, G.F., Lavergne, S., Rebelo, A.G., 2004, Relating plant traits and species distributions along bioclimatic gradients for 88 Leucadendron species in the Cape Floristic Region, Ecology, 85: $1688-1699$.

11. Matthews, L.J., 2002, The Protea book: a guide to cultivated Proteaceae, New Zealand, Canterbury University Press.

12. Pharmawati, M., Yan, G., Finnegan, P.M., 2005, Molecular variation and fingerprinting of Leucadendron cultivars (Proteaceae) by ISSR markers, Annals of Botany, 95 (7):1163-1170. doi:10.1093/aob/ mci127.

13. South African National Biodiversity Institute Red List 2020 version for genus Leucadendron http://redlist.sanbi.org/genus.php?genus=794

14. Mankga., L.T., Yessoufou, K., 2017, Factors driving the global decline of cycad diversity. AoB PLANTS. 9, https://doi.org /10.1093/aobpla/plx022.

15. Rodrigues, S.L., Pilgrim, J.D., Lamoreux, J.F., Hoffmann, M., Brooks T.M.. 2006, The value of IUCN red list for Conservation, Trends in Ecology and Evolution, 21 (2): 71-76.

\section{EVALUAREA STATUSULUI CONSERVATIV, A AMENINȚĂRILOR ȘI TRENDULUI POPULAȚIONAL AL GENULUI LEUCADENDRON, ENDEMIC PENTRU REGIUNEA CAPULUI DIN AFRICA DE SUD}

\section{(Rezumat)}

Scăderea numărului de specii endemice are un impact semnificativ asupra pierderii biodiversității globale. Trebuie depuse eforturi suplimentare pentru a proteja în continuare speciile endemice de actuala criză globală a dispariției. Acest studiu a evaluat starea actuală de conservare, factorii responsabili pentru riscul de extincție și tendințele populațiilor de Leucadendron, un gen de plante endemic pentru Regiunea Capului din Sudul Africii. Pentru acest studiu s-a folosit Lista Roșie a ISABN (Institutul Sud African de Biodiersitate Naţională). Rezultatele au arătat că peste 50\% dintre taxonii acestui gen sunt amenințați, iar majoritatea speciilor sunt expuse la distrugerea habitatului și prezența speciilor invazive. De asemenea, s-a descoperit că două specii ale genului sunt actualmente extincte, ceea ce presupune că genul de plante este supus unui risc ridicat de extincție. Ar trebui depuse mai multe eforturi, cum ar fi proiectarea unor metode de a controla forțele responsabile pentru riscul de extincție a taxonilor acestui gen, pentru a preveni eliminarea completă a acestora în viitor. 